\title{
PERFIL DE HABILIDADES DE COMUNICAÇÃO DE DOIS IRMÃOS COM A SÍNDROME ALCOÓLICA FETAL
}

\section{Communicative profile in two siblings with Fetal Alcohol Syndrome}

\author{
Roberta Garcia ${ }^{(1)}$, Natalia Freitas Rossi ${ }^{(2)}$, Célia Maria Giacheti ${ }^{(3)}$
}

\section{RESUMO}

Objetivo: caracterizar o perfil de habilidades de comunicação e o desempenho nos subtestes do Teste Illinois de Habilidades Psicolingüísticas (ITPA) de dois irmãos com diagnóstico da Síndrome Alcoólica Fetal (SAF): S1, sexo feminino, 16 anos e S2, sexo masculino, 8 anos, alunos de escola especial. Métodos: realizou-se a avaliação fonoaudiológica clínica, complementada pela aplicação do ITPA. Resultados: dados de avaliação clínica com S1 mostraram prejuízo grave de compreensão oral, não sendo capaz de seguir instruções (verbal e gestual) simples. Também não se observou uso de recursos gestuais ou orais, por parte da mesma para se comunicar. Devido ao nível de comprometimento, não foi possível obter dados relativos aos desempenhos amostrados no ITPA. Dados de avaliação clínica com S2 mostraram resultados bastante diferentes. S2 utiliza preferencialmente a fala para se comunicar, responde perguntas, mantendo o tema e é capaz de seguir instruções verbais simples. Entretanto, observou-se desempenho inferior ao esperado para idade em situações que requeriam narrativa de fatos e eventos, indicando prejuízos em aspectos sintáticos e semânticos da linguagem. No ITPA obteve pontuação inferior a idade cronológica em quase todos os subtestes. O pior desempenho foi observado nos subtestes que envolviam habilidades perceptivas auditivas. Conclusões: esses resultados indicam que, apesar de ambos terem o mesmo diagnóstico genético e viverem sob as mesmas condições familiares, o perfil fonoaudiológico difere. Pode-se especular que prejuízos mais graves em habilidades comunicativas de $\mathrm{S} 1$ podem estar relacionados a níveis mais elevados de exposição ao álcool durante etapas críticas do desenvolvimento fetal.

DESCRITORES: Síndrome Alcoólica Fetal; Comunicação; Linguagem; Aprendizagem

\section{INTRODUÇÃO}

Há mais de 30 anos pesquisadores descreveram as alterações físicas e comportamentais presentes em crianças cujas mães apresentavam antecedentes do uso de álcool durante a gestação. O conjunto

(1) Fonoaudióloga; Bolsista de Apoio Técnico (CNPq) do Setor de Fonogenética do Hospital de Reabilitação de Anomalias Craniofaciais da Universidade de São Paulo - Campus de Bauru (Processo número 373556/2006-3).

(2) Fonoaudióloga; Aluna regular do Programa de Pós-Graduação em Ciências Biológicas - Área de Concentração: Genética, Nível Doutorado, do Instituto de Biociências, Universidade Estadual Paulista - Campus de Botucatu, Bolsista CNPq.

(3) Fonoaudióloga; Livre Docente do Departamento de Fonoaudiologia da Faculdade de Filosofia e Ciências da Universidade Estadual Paulista - Campus de Marília e Orientadora do Programa de Pós-Graduação em Ciências Biológicas - Área de Concentração: Genética, do Instituto de Biociências da Universidade Estadual Paulista Campus de Botucatu. destas características culminou na descrição desta condição, denominada "Síndrome Alcoólica Fetal (SAF)" ${ }^{1,2}$.

De forma geral a literatura mostra que o fenótipo desta condição é definido pela tríade sintomática que inclui: dismorfismos faciais, prejuízo no crescimento pré e pós-natal e malformações do Sistema Nervoso Central (SNC), acrescido ao histórico materno para o uso de álcool durante a gestação. $O$ diagnóstico da SAF é atribuído aos casos em que todas essas alterações estão presentes. Porém, algumas das características físicas e comportamentais típicas da SAF podem estar presentes, acrescidas do histórico materno para o uso de álcool na gestação e que não definem o diagnóstico da SAF. Neste caso, o quadro é conhecido como "Desordem Neurodesenvolvimental Relacionada ao Álcool" 3.

A etiologia da SAF é ambiental e sua severidade advém da combinação da ingestão de álcool pela mãe com algumas modalidades de fatores de risco, tais como quantidade e freqüência com que o álcool 
foi consumido pela gestante, período da gestação em que a mãe ingeriu a substância, idade materna e seus aspectos genéticos, nutricionais e físicos, o uso concomitante de tabaco e/ou outras drogas, fatores socioeconômicos, psicológicos, familiares e tolerância da mãe e do feto aos possíveis efeitos teratogênicos do álcool ${ }^{4-6}$.

A prevalência da SAF no Brasil foi estimada em torno de 1/1000 nascidos vivos. Porém, sugere-se que este dado deva estar subestimado, uma vez que é uma afecção de difícil diagnóstico, não é de notificação compulsória e o consumo de bebidas alcoólicas pelas gestantes no Brasil é significativo ${ }^{7}$.

A maior parte das variações nos achados quanto à prevalência da SAF pode ser explicada devido à dificuldade e variação de estratégias diagnósticas dos estudos realizados. Além disso, estimativas sugerem que aproximadamente $5 \%$ das mulheres alcoolistas, que ingeriram bebidas alcoólicas cronicamente, durante a gestação terá uma criança com a SAF ${ }^{8}$.

As manifestações que constituem o fenótipo desta condição estão presentes ao longo da vida desses indivíduos, o que acarreta prejuízos sociais e ocupacionais, tornando-o dependente da assistência da família e de vários profissionais de saúde ${ }^{9}$.

\section{Fenótipo da SAF: aspectos faciais e neurodesenvolvimentais}

As características faciais representam um dos principais sinais clínicos que torna possível o diagnóstico precoce da síndrome, somado às manifestações comportamentais ${ }^{7}$.

Dentre as principais características faciais observa-se hemiface achatada, fissuras palpebrais curtas, ptose, narinas antevertidas, filtro nasal apagado e lábio superior fino e liso. Além das características faciais, podem-se encontrar outras alterações como anomalias digitais, deficiência mental, alterações nas habilidades de comunicação, lingüística e de aprendizagem, além de alterações comportamentais e nos aspectos sócio-interacionais ${ }^{4,5,10-14}$.

Há uma possível correlação entre os dismorfismos faciais e a incidência de alterações da linha média, sugerindo que quanto mais graves forem esses dismorfismos, maiores são as alterações do Sistema Nervoso Central (SNC), envolvendo a linha média ${ }^{15}$.

Alterações estruturais nos lóbulos frontal e parietal - mais especificamente a região perisilviana - no cerebelo, gânglios basais e no corpo caloso, são as mais comuns de serem encontradas, o que justifica a presença de alterações nas habilidades da linguagem, na aprendizagem, nas funções cognitivas e também motoras ${ }^{16-18}$, com comprometimento da coordenação bimanual ${ }^{19}$.

\section{Aspectos audiológicos, cognitivos e lingüísticos na SAF}

Quatro tipos de desordens audiológicas foram relatados em portadores da SAF: atraso no desenvolvimento da função auditiva, perda auditiva do tipo neurossensorial, perda auditiva do tipo condutiva e perda auditiva central ${ }^{20}$. Devido à recorrência de otite média crônica, muitas vezes justificada pela presença de fissura lábio-palatina, torna-se comum o quadro de perda auditiva do tipo condutiva em indivíduos com a síndrome alcoólica fetal ${ }^{21}$.

Prejuízos na fala e atraso na aquisição e desenvolvimento da linguagem, receptiva e expressiva, são manifestações comuns aos indivíduos com a SAF, sendo a sintaxe um dos componentes mais comprometidos ${ }^{22,23}$.

Várias pesquisas comprovaram a presença de dificuldades de memória, envolvendo prejuízos tanto no armazenamento quanto na evocação da informação verbal, o que justificaria as dificuldades na aprendizagem global desses indivíduos 12,13,24,25, incluindo o aprendizado de conceitos lógico-matemáticos e também sócio-comunicativos ${ }^{26-28}$. O tempo aumentado para o processamento da informação ${ }^{29,30}$, somado a dificuldade de atenção e o comportamento hiperativo/impulsivo, sugere ser um dos principais fatores relacionados à dificuldade de aprendizagem desses indivíduos ${ }^{31}$.

No Brasil, ainda são escassas pesquisas direcionadas a investigação do perfil de habilidades de linguagem em pacientes com SAF. Sendo assim, o objetivo deste estudo é caracterizar o perfil de habilidades de comunicação e o desempenho nos subtestes do Teste Illinois de Habilidades Psicolingüísticas (ITPA) de dois irmãos com diagnóstico da SAF: $\mathrm{S} 1$, sexo feminino, 16 anos e $\mathrm{S} 2$, sexo masculino, 8 anos, ambos matriculados em escola especial.

\section{MÉTODOS}

Para caracterização do perfil de comunicação dos irmãos com SAF foi realizada avaliação fonoaudiológica clínica que incluiu: avaliação das habilidades comunicativas, que analisou a intencionalidade e o uso de recursos comunicativos verbais e nãoverbais utilizados pelos sujeitos; a linguagem oral considerando as habilidades fonológicas, sintáticas, semânticas e pragmáticas e a linguagem escrita.

Os irmãos foram avaliados por meio de conversa espontânea e semidirigida. Quando não foi possível utilizar estas estratégias, a avaliação baseou-se na observação dos recursos comunicativos utilizados pelo sujeito submetido a contexto pragmático de uso da linguagem.

A habilidade fonológica foi avaliada pela Avaliação Fonológica da Criança (AFC) ${ }^{32}$ para carac- 
terização do inventário fonológico e dos processos fonológicos utilizados pelos sujeitos.

Foi realizada a aplicação do Teste Illinois de Habilidades Psicolingüísticas (ITPA) ${ }^{33,34} \mathrm{com}$ a finalidade de caracterizar o desempenho em tarefas viso-motoras e auditivo-vocais, incluindo aspectos lingüísticos e memória. Este teste engloba 12 subtestes que avaliam a recepção auditiva e visual, a memória seqüencial auditiva e visual, a associação auditiva e visual, a "closura" auditiva, visual e gramatical, a expressão verbal e manual e a combinação de sons.

Para caracterizar o perfil audiológico dos sujeitos, foram realizados três procedimentos: otoscopia, realizada por médico otorrinolaringologista; medidas de imitância acústica e audiometria tonal limiar.

Este estudo foi aprovado pelo Comitê de Ética em Pesquisa da Faculdade de Filosofia e Ciências de Marília - UNESP, sob o protocolo de $n^{\circ}$ 2715/2003. A participação dos sujeitos foi aprovada mediante assinatura do Termo de Consentimento Livre e Esclarecido elaborado para fins específicos desta pesquisa, segundo resolução n 196/96.

\section{RESULTADOS}

O heredograma (Figura 1) ilustra a história familial de dois irmãos com antecedentes positivos para alcoolismo entre os pais (II-1 e II-2) e também para a progenitora materna (I-1). A mãe dos sujeitos confirmou o uso de álcool etílico nas três gestações dos filhos III-1(S1); III-2 e III-3(S2). A avaliação genética do sujeito III-2 mostrou sinais do efeito do álcool na gestação (hiperatividade e distúrbio de aprendizagem), porém não apresentou o diagnóstico da síndrome alcoólica fetal.

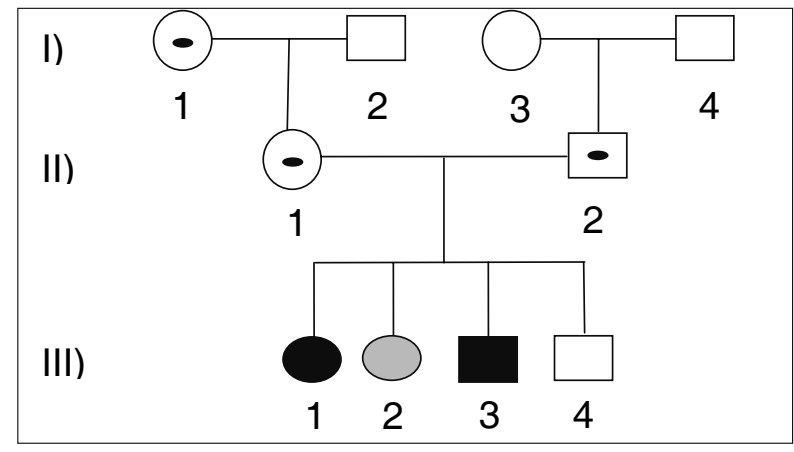

Legenda:

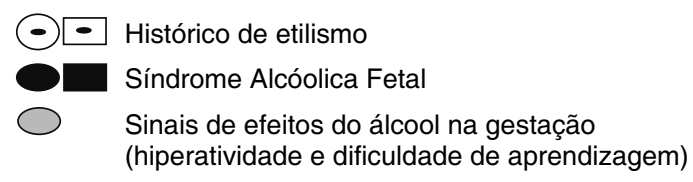

Figura 1 - Heredograma I

\section{Descrição de caso}

Sujeito 1

L.L.P, 16 anos de idade, primeira filha do casal, mãe referiu ingestão de álcool na gestação (aproximadamente duas garrafas de destilado/ dia). O sujeito nasceu de parto normal, pesando $2.600 \mathrm{Kg}$, com o uso de fórceps e a ocorrência de cianose ao nascimento. Há relato de atraso neuropsicomotor, com controle cervical por volta do $5^{\circ}$ mês, início da marcha aos 2 anos e não apresenta controle dos esfíncteres anal e vesical. Freqüenta o ensino especial e não há relatos de emissão de palavras, apenas de sons guturais e de comportamentos restritos e repetitivos como balanceio de tronco e cabeça, tendência ao isolamento e agitação.

\section{Sujeito 2}

A.S.P, 8 anos e 7 meses de idade, terceiro filho do casal, mãe também confirmou a ingestão de álcool etílico nas mesmas proporções da primeira gestação. Nasceu de parto normal, com 1.600 $\mathrm{Kg}$, caracterizando baixo peso ao nascimento. A informante não soube precisar as etapas do desenvolvimento neuropsicomotor do sujeito, referiu que sentou aos 8 meses de idade e apresentou controle dos esfíncteres anal e vesical aos três anos e meio de idade. Apresentou início da fala por volta dos 2 anos de idade e por algum tempo se comunicava utilizando palavras isoladas e restritas. A informante referiu que o sujeito apresenta comportamentos de agitação, e queixou-se de problemas no aprendizado escolar.

O diagnóstico da SAF para ambos os casos foi realizado por médico geneticista do HRAC, a partir do histórico gestacional positivo para o uso de álcool e a identificação dos sinais clínicos da síndrome.

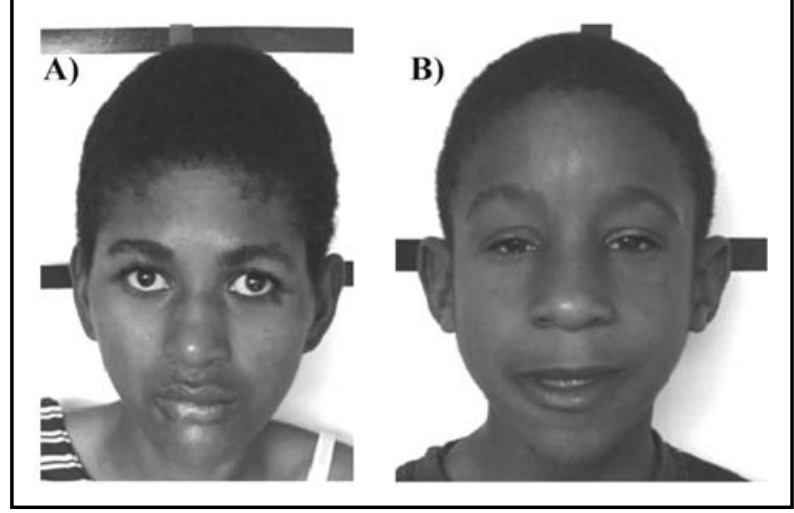

Figura 2 - Observar as características faciais da Síndrome Alcoólica Fetal dos irmãos. Figura 2A: S1 apresenta face plana, microcefalia, filtro nasal apagado, ponte nasal rebaixada, microftalmia, micrognatia. Figura 2B: S2 apresenta microcefalia, lábio superior fino e achatado, filtro nasal apagado, ponta nasal rebaixada microftalmia e micrognatia 
Ambos apresentaram característica facial típica da SAF, embora variável entre eles (Figura 2A e 2B). O perímetro cefálico do $S 1$ foi $0,54 \mathrm{~cm}$, dentro do esperado para o sexo e idade. Para o S2 a medida do perímetro cefálico foi $0,49 \mathrm{~cm}$, abaixo do 2DP. Em relação à medida estatural, $\mathrm{S} 1$ não pode ser avaliado devido a problemas comportamentais e o S2 apresentou estatura de 1,20m correspondente ao esperado para a idade.

A partir da avaliação fonoaudiológica clínica foi possível realizar a caracterização dos achados referentes às habilidades comunicativas, de linguagem oral e escrita além da caracterização dos achados audiológicos.

\section{Habilidades Comunicativas}

Durante o processo de interação comunicativa foi possível observar desempenho variável entre os irmãos quanto à intencionalidade e uso de recursos comunicativos apresentados pelos mesmos. S1 demonstrou freqüência restrita de atos comunicativos se comparado a S2, assim como no uso de sinalizadores atencionais como contato visual e recursos de feedback que estiveram mais prejudicados para S1. O uso da linguagem oral como recurso comunicativo, esteve muito prejudicado para ambos, novamente mais grave para S1 que apresentou ausência de emissão oral. Foi possível constatar ininteligibilidade de fala para S2 e dificuldade compreensão oral para ambos, sendo verificado que S1 apresentou comprometimento para compreender ordens simples e S2 ordens de média complexidade.

\section{Linguagem Oral e Escrita}

A partir da avaliação da linguagem oral, considerando aspectos expressivos e receptivos, foi observada ausência de emissão oral e prejuízo significativo no reconhecimento e execução de ordens simples para S1.

A ausência de amostra de fala impossibilitou que fosse realizada a caracterização do inventário fonológico do S1. Também não foi realizada a aplicação de provas de leitura e escrita para este sujeito, uma vez que o mesmo não obteve nível lingüístico para tais habilidades. Desta forma, verificou-se que S1 apresentou comprometimento significativo das habilidades sintática, semântica, fonológica e pragmática da linguagem, caracterizando quadro de Distúrbio de Linguagem.

Dificuldades para compreender e estruturar a narrativa oral, evidenciando falhas de correlação lexical e no uso de elementos gramaticais, foram identificadas no desempenho do S2. Notou-se que o mesmo apresentou narrativa curta e simplista do ponto de vista estrutural e quanto ao uso das habi- lidades lingüísticas; com uso de estruturação frasal simples e prejuízos quanto à organização interna dos elementos gramaticais e coerência semântica. O sujeito demonstrou habilidade para manter o tema abordado, porém, com prejuízos significativos no que se refere à troca de turno e a escolha e introdução de novos temas. Em relação à compreensão oral, foi possível verificar habilidade preservada para compreender ordens simples com prejuízos para interpretar enunciados com estruturação sintática complexa e de conteúdo que requeriam a compreensão de ambigüidades e processamento não-literal da informação. Quanto à habilidade pragmática o sujeito mostrou capacidade de simbolizar ações e utilizar os objetos funcionalmente, porém com dificuldades quanto ao uso da linguagem oral, como principal recurso de comunicação. O desempenho apresentado pelo sujeito apontou para comprometimentos significativos de natureza lingüística-pragmática, uma vez que, tanto o uso de recursos comunicativos quanto o domínio de competências lingüísticas estiveram prejudicadas.

A presença de prejuízo fonológico pode ser constatada, junto às demais dificuldades de linguagem já relatadas, uma vez que o S2 apresentou inteligibilidade de fala alterada, o que dificultou caracterização do inventário fonológico a partir do instrumento proposto. A ininteligibilidade de fala esteve associada à co-ocorrência de processos fonológicos, envolvendo principalmente os processos de natureza silábica.

Verificaram-se ainda dificuldades para responder as tarefas que requeriam compreensão, planejamento e execução de situação-problema, incluindo comprometimento para reconhecer relações numéricas, mesmo com símbolos concretos. Quanto à linguagem escrita, o paciente demonstrou reconhecimento e escrita de grafemas isolados e de numerais, assistematicamente. Dificuldades viso-espacial puderam ser identificadas em situação de escrita com ocorrência de inversões de letras e linhas flutuantes. Este desempenho revelou dificuldades nas habilidades de raciocínio lógico e matemático e no aprendizado das regras de decodificação grafema-fonema. Diante do exposto, o diagnóstico fonoaudiológico do S2 é Distúrbio de Aprendizagem.

De um modo geral, nota-se que houve variabilidade quanto ao grau de comprometimento das manifestações de linguagem observadas entre os irmãos, o que justificou o diagnóstico de Distúrbio de Linguagem para o S1 e Distúrbio de Aprendizagem para o S2. 


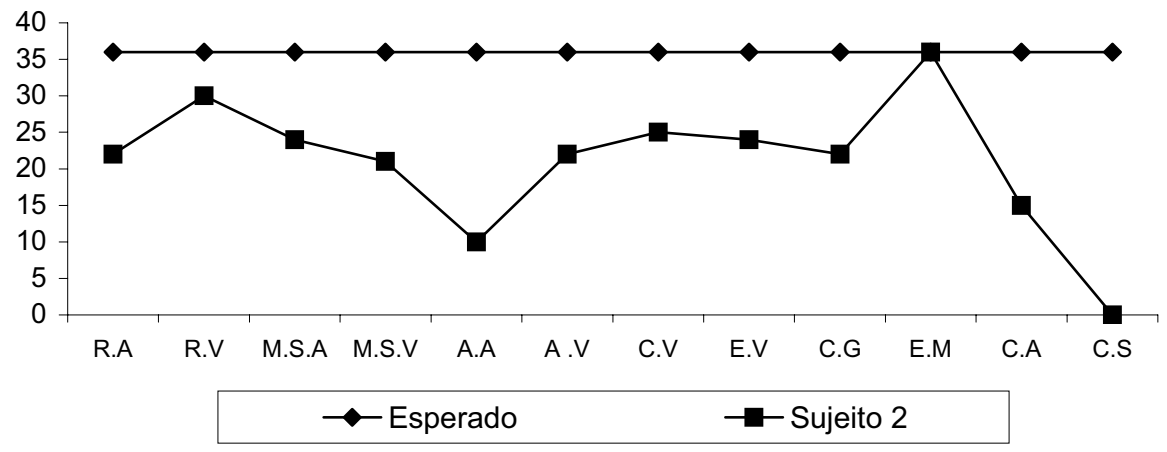

Legenda:

R.A - recepção auditiva

R.V - recepção visual

M.S.V - memória seqüencial visual

M.S.A - memória seqüencial auditiva

A.V - associação visual

A.A - associação auditiva
C.V - closura visual

E.V - expressão verbal

C.G - closura gramatical

E.M - expressão manual

C.A - closura auditiva

C.S - combinação de sons

Figura 3 - Representação Gráfica do Escore Escalar do Sujeito 2 comparado ao desempenho esperado a idade cronológica

Avaliação Fonoaudiológica Complementar: Teste Illinois de Habilidades Psicolingüísticas ITPA

O ITPA não pode ser pontuado para a análise do desempenho de $S 1$, uma vez que o mesmo não atingiu o escore base para prosseguimento dos subtestes fornecidos pelo ITPA. Tal ocorrência deve-se ao significativo prejuízo de compreensão oral e também às alterações comportamentais, como prejuízo atencional e agitação.

O desempenho de S2, no ITPA, mostrou escore escalar abaixo do esperado para idade, para todos os subtestes avaliados com exceção para o subteste de expressão manual (Figura 3 ). A análise do desempenho do sujeito mostrou que os subtestes mais prejudicados foram os que envolviam as habilidades auditivas se comparadas às visuais. Desta forma, foi possível verificar que S2 apresentou idade psicolingüística significativamente abaixo de sua idade cronológica, exceto para o subteste de expressão manual.

\section{Avaliação audiológica}

Dentre os procedimentos de avaliação audiológica propostos, S1 submeteu-se apenas a realização da otoscopia, a qual apresentou resultado normal. Não foi possível realizar as demais avaliações devido à dificuldade de compreensão em seguir instruções e agitação constante do sujeito, impossibilitando a colocação de sondas e de fones de ouvido, imprescindíveis à realização dos exames.

S2 realizou todo o procedimento audiológico, apresentando avaliação otoscópica normal, perda auditiva do tipo condutiva de grau leve bilateralmente e curva timpanométrica tipo $\mathrm{C}$ bilateralmente, com ausência dos reflexos acústicos contralaterais também bilateralmente.

\section{DISCUSSÃO}

A divulgação das alterações fonoaudiológicas relacionadas à SAF foram abordadas de forma mais específica a partir da década de 90 , quando os estudos na área se intensificaram e ganharam credibilidade por parte da comunidade científica. Porém, essas pesquisas sobre o perfil de habilidades de linguagem em pacientes com SAF ainda são escassas, principalmente na literatura brasileira.

Desta forma, a proposta do presente estudo de realizar a caracterização do perfil de habilidades de comunicação de dois irmãos portadores da síndrome alcoólica fetal, vem a somar os demais achados da literatura. A partir dos casos apresentados, é evidente a gama de alterações fonoaudiológicas, tanto nos aspectos da linguagem oral como ao aprendizado da leitura e escrita, que estão associadas aos sujeitos avaliados com diagnóstico de SAF.

Considerando que o consumo de álcool na população vem aumentando, principalmente entre as mulheres, este componente ambiental somado aos fatores de pré-disposição genética contribui para o aumento de casos na população e também de recorrência familial ${ }^{8}$, condição esta apresentada por este trabalho. Desta forma a SAF está se tornando um problema de saúde pública, embora seja 
totalmente passível de prevenção, pela não ingestão de álcool durante a gestação.

O diagnóstico da SAF ainda é bastante difícil, uma vez que requer o reconhecimento dos principais sinais clínicos somados ao histórico positivo de exposição ao álcool intra-útero. A variabilidade quanto ao grau de comprometimento tanto dismórfico quanto comportamental também são fatores que dificultam o reconhecimento desta condição. Este estudo mostrou que embora os irmãos tenham apresentado os principais sinais clínicos da SAF (Figura 2A e 2B) como a face plana, filtro nasal apagado, ponte nasal rebaixada e lábio superior fino e achatado; microftalmia; micrognatia e anomalias digitais ${ }^{5,14}$, a variabilidade de manifestação esteve presente entre eles.

A variabilidade de manifestações também foi encontrada no que concerne às habilidades de comunicação dos irmãos. O caso mais grave no que diz respeito ao aspecto lingüístico-cognitivo foi S1, embora seja S2 que apresente os dismorfismos faciais mais fortemente relacionados a SAF. $O$ estudo sugere que os sinais faciais podem não estar diretamente relacionados ao grau de comprometimento dos aspectos neurodesenvolvimentais.

A descrição dos irmãos com a SAF apontou grave comprometimento nas habilidades de linguagem/aprendizagem, bem como de problemas comportamentais como parte do fenótipo desta síndrome. Este achado corroborou pesquisas em que foi relatado que crianças cujas mães ingeriram álcool durante a gravidez podem sofrer vários graus de anomalias neurológicas, cognitivas e comportamentais ${ }^{27,28,35}$.

Quanto às habilidades de leitura e escrita, este trabalho evidenciou que ambos os sujeitos apresentaram alterações significativas, sendo estas prejudiciais ao processo de aprendizagem da leitura e da escrita. Alguns estudos publicados na área também encontraram dificuldades quanto ao desempenho das habilidades acadêmicas nos portadores da $\mathrm{SAF}^{28,31}$.

O ITPA permitiu que a avaliação fonoaudiológica fosse complementada a partir do perfil de habilidades psicolingüísticas, o qual também só pôde ser obtido pelo desempenho do S2, uma vez que o S1 não atingiu escore base para continuidade da aplicação dos subtestes.

De um modo geral, o desempenho do sujeito esteve mais alterado nos subtestes que envolviam as habilidades auditivas em relação as visuais. Este resultado corrobora a possibilidade de prejuízos no sistema auditivo central ${ }^{20}$ relacionado com o processamento da linguagem, o que justifica estudos futuros com esta população.

Sendo o canal visual o mais preservado nos portadores da SAF, sugere-se que este seja eleito como via preferencial de acesso ao trabalho com o processo atencional e de memória, essenciais para o desenvolvimento da aprendizagem.

A literatura mostrou que a perda auditiva do tipo condutiva pode estar presente nos indivíduos com a $\mathrm{SAF}^{20}$, assim como foi identificado no S2. Ressaltase, porém, que este achado não sugere ser o principal fator responsável pelos prejuízos de linguagem apresentados pelo mesmo.

\section{CONCLUSÕES}

Os resultados mostraram que apesar de ambos terem o diagnóstico da SAF e viverem sob as mesmas condições ambientais, o perfil de comunicação diferiu entre os irmãos. Pode-se especular que prejuízos mais graves em habilidades comunicativas do $\mathrm{S} 1$, podem estar relacionados a níveis mais elevados de exposição ao álcool durante etapas críticas do desenvolvimento fetal.

Perante a variabilidade do grau de comprometimento encontrado entre os sujeitos avaliados, tanto no que se refere ao desempenho das habilidades de comunicação, quanto ao desempenho no ITPA do $S 1$, sugere-se que estudos complementares venham aprofundar os conhecimentos relacionados a esta condição ambiental e genética, a fim de estabelecer possíveis associações entre fatores de exposição pré-natal ao álcool e fatores de pré-disposição genética, com os diferentes graus de comprometimento fonoaudiológico dos sujeitos avaliados.

Destaca-se a importância de estudos mais abrangentes com esta população, referindo-se tanto ao aumento da casuística, quanto a investigações de habilidades cognitivas e lingüísticas específicas, para melhor compreender a interferência do perfil de comunicação desses indivíduos junto aos aspectos sócio-comunicativos e educacionais.

\section{AGRADECIMENTOS}

Ao Conselho Nacional de Desenvolvimento Científico e Tecnológico - CNPq - pelo auxílio financeiro concedido durante a realização deste trabalho. 


\begin{abstract}
Purpose: to characterize the communication profile and the performance in the subtests of Illinois Test of Psycholinguistic Abilities (ITPA) of two siblings with Fetal Alcohol syndrome (FAS): S1, female 16 years and S2, male, 8 years and both of special school students. Methods: clinical evaluation of language abilities was carried out and complemented by the ITPA test. Results: data of clinical evaluation with $\mathrm{S} 1$ showed verbal understanding impairment. S1 was not capable to follow simple instructions (verbal and gestual) and the use of verbal and gestual resources communication was not also observed. Due to the compromising level, it was not possible to obtain relative data to the ITPA test. Quite different results was observed in the clinical evaluation of S2, considering that he was able to make questions, maintaining the theme and to follow simple verbal instructions. However, low performance was observed for age in situations that requested narrative of facts and events that shown syntactic and semantic impairments. Scale score obtained by S2 in the ITPA subtests revealed low performance in auditory and visual abilities. Conclusions: although the siblings have the same diagnosis and live under the same environmental conditions, the communication profile was significantly different. It can be speculated that more serious impairments in communicative abilities of S1 can be related to higher levels of exposure to alcohol during critical stages of the fetal development.
\end{abstract}

KEYWORDS: Fetal Alcohol Syndrome; Communication; Language; Learning

\section{REFERÊNCIAS}

1. Lemoine $P$, Harousseau $H$, Borteyru PJ, Menuet CJ. Les enfants de parents alcooliques: anomalies observées à propos de 127 cas. Ouest Méd. 1968; 21:476-82.

2. Jones KL, Smith DW, Ulleland CN, Streissguth $P$. Pattern of malformation in offspring of chronic alcoholic mothers. Lancet. 1973; 1(7815):1267-71.

3. Stratton K, Howe C, Battaglia F, editors. Fetal alcohol syndrome: diagnosis, epidemiology, prevention and treatment. Washington: National Academy Press; 1996. p. 4-21.

4. Gardner J. Living with a child with fetal alcohol syndrome. Am J Matern Child Nurs. 2000; 25:252-7.

5. Roccella M, Testa D. Fetal alcohol syndrome in developmental age: neuropsychiatric aspects. Minerva Pediatr. 2003; 55(1):63-74.

6. May PA, Fiorentino D, Phillip Gossage J, Kalberg WO, Eugene Hoyme $\mathrm{H}$, Robinson LK, et al. Epidemiology of FASD in a province in Italy: prevalence and characteristics of children in a random sample of schools. Alcohol Clin Exp Res. 2006; 30(9):1562-75.

7. Grinfeld H, Segre CAM, Chadi G, Goldenberg S. $\mathrm{O}$ alcoolismo na gravidez e os efeitos na prole. Rev Paul Pediatr. 2000; 18:41-9.

8. Burd L, Cotsonas-Hassler TM, Martsolf JT, Kerbeshian J. Recognition and management of fetal alcohol syndrome. Neurotoxicol Teratol. 2003; 25(6):681-8.
9. Merrick J, Merrick E, Morad M, Kandel I. Fetal alcohol syndrome and its long-term effects. Minerva Pediatr. 2006; 58(3):211-8.

10. Streissguth AP, Barr HM, Kogan J, Bookstein FL. Understanding the occurrence of secondary disabilities in clients with fetal alcohol syndrome (FAS) and fetal alcohol effects (FAE) [thesis]. Seattle(USA): University of Washington Publication Services; 1996.

11. Shprintzen RJ. Genetics, syndromes, and communication disorders. San Diego: Singular; 1997. $315 \mathrm{p}$.

12. Mattson SN, Schoenfeld AM, Riley EP. Teratogenic effects of alcohol on brain and behavior. Alcohol Res Health. 2001; 25(3):185-91.

13. O'Malley KD, Nanson J. Clinical implications of a link between fetal alcohol spectrum disorder and attention-deficit hyperactivity disorder. Can J Psychiatry. 2002; 47(4):349-54.

14. Suzuki K. Adult fetal alcohol syndrome (FAS) with various neuropsychiatric symptoms. Nihon Arukoru Yakubutsu Igakkai Zasshi. 2004; 39(5):474-81.

15. Swayze VW, Johnson VP, Hanson JW, Piven J, Sato $Y$, Giedd JN, et al. Magnetic resonance imaging of brain anomalies in fetal alcohol syndrome. Pediatrics. 1997; 99(2):232-40.

16. Sowell ER, Thompson PM, Mattson SN, Tessner KD, Jernigan TL, Riley EP, et al. Regional brain shape abnormalities persist into adolescence after heavy prenatal alcohol exposure. Cereb Cortex. 2002; 12(8):856-65.

17. Bookstein FL, Streissguth AP, Sampson PD, Connor PD, Barr HM. Corpus callosum shape and 
neuropsychological deficits in adult males with heavy fetal alcohol exposure. Neuroimage. 2002; 15(1):233-51.

18. Riley EP, McGee CL, Sowell ER. Teratogenic effects of alcohol: a decade of brain imaging. Am J Med Genet C Semin Med Genet. 2004; 127(1):35-41.

19. Roebuck-Spencer TM, Mattson SN, Marion SD, Brown WS, Riley EP. Bimanual coordination in alcohol-exposed children: role of the corpus callosum. $\mathrm{J}$ Int Neuropsychol Soc. 2004; 10(4):536-48.

20. Church MW, Abel EL. Fetal alcohol syndrome: hearing, speech, language, and vestibular disorders. Obstet Gynecol Clin North Am. 1998; 25(1):85-97.

21. Cone-Wesson B. Prenatal alcohol and cocaine exposure: influences on cognition, speech, language, and hearing. J Commun Disord. 2005; 38(4):279-302.

22. Becker M, Warr-Leeper GA, Leeper HA. Fetal alcohol syndrome: a description of oral motor, articulatory, short-term memory, grammatical, and semantic abilities. J Commun Disord. 1990; 23(2):97-124. 23. Carney LJ, Chermak GD. Performance of american indian children with fetal alcohol syndrome on the test of language development. J Commun Disord. 1991; 24(2):123-34.

24. Mattson SN, Roebuck TM. Acquisition and retention of verbal and nonverbal information in children with heavy prenatal alcohol exposure. Alcohol Clin Exp Res. 2002; 26(6):875-82.

25. Willford JA, Richardson GA, Leech SL, Day NL. Verbal and visuospatial and memory function in children with moderate prenatal alcohol exposure. Alcohol Clin Exp Res. 2004; 28(3):497-507.

26. Rasmussen $C$. Executive functioning and working memory in fetal alcohol spectrum disorder. Alcohol Clin Exp Res. 2005; 29(8):1359-67.
27. Schonfeld AM, Mattson SN, Riley EP. Moral maturity and delinquency after prenatal alcohol exposure. J Stud Alcohol. 2005; 66(4):545-54.

28. Howell KK, Lynch ME, Platzman KA, Smith $\mathrm{GH}$, Coles CD. Prenatal alcohol exposure and ability, academic achievement, and school functioning in adolescence: a longitudinal follow-up. J Pediatr Psychol. 2006; 31(1):116-26.

29. Burden MJ, Jacobson SW, Jacobson JL. Relation of prenatal alcohol exposure to cognitive processing speed and efficiency in childhood. Alcohol Clin Exp Res. 2005; 29(8):1473-83.

30. Simmons RW, Thomas JD, Levy SS, Riley EP. Motor response selection in children with fetal alcohol spectrum disorders. Neurotoxicol Teratol. 2006; 28(2):278-85.

31. Kodituwakku P, Coriale G, Fiorentino D, Aragon AS, Kalberg WO, Buckley D, et al. Neurobehavioral characteristics of children with fetal alcohol spectrum disorders in communities from Italy: preliminary results. Alcohol Clin Exp Res. 2006; 30(9):1551-61.

32. Yavas M, Hernandorena CLM, Lamprecht RR. Avaliação fonológica da criança. Porto Alegre: Artes Médicas; 1992.148 p.

33. Kirk SA, McCarthy JJ. The Illinois test of psycolinguistic abilities. Urbana: University Illinois Press; 1968. $136 \mathrm{p}$.

34. Bogossian MADS, Santos MJ. Manual do examinador: teste de habilidades psicolingüísticas. Rio de Janeiro: EMPSI; 1977. 64 p.

35. Sowell ER, Thompson PM, Mattson SN, Tessner KD, Jernigan TL, Riley EP, et al. Voxel-based morphometric analyses of the brain in children and adolescents prenatally exposed to alcohol. Neuroreport. 2001; 12(3):515-23.

Endereço para correspondência:

Rua Amazonas, 420

Araraquara - SP

CEP: 14811-066

Tel: (16) 33373041 / (16) 81287387

E-mail: roberttagarcia@ig.com.br 